\title{
Automatic Pneumatic Power Based Object Sorting System by Using MITSUBISHI PLC
}

\author{
Mr.P.Choudesh Varma ${ }^{1}$ and Mr.G.Venkateswarlu ${ }^{2}$ \\ ${ }^{1}$ CVR College of Engineering, Department of EIE., Ibrahimpatan, R.R.District, A.P., India \\ Email: choudesh82@gmail.com \\ ${ }^{2}$ CVR College of Engineering, Department of EIE., Ibrahimpatan, R.R.District, A.P., India \\ Email: venkigummadilli@gmail.com
}

\begin{abstract}
This paper presents the Automatic Pneumatic Power Based Object Sorting System by Using MITSUBISHI PLC. PLC stands for Programmable Logic controllers. The purpose of the project is to provide low power object sorting with pneumatic pushing system. This project or idea is the basic need of almost every type of manufacturing and production unit today like Food/ Beverage, Metal, Mining, Power, Textile, Automobile, Chemical, Pharmacy, soft \& hard drink manufacturing industries etc are the few examples where we see the automation today. The main objective of the project mainly follows two sequential steps. First step is to detect the size of the object by using IR detectors. IR detectors are placed at three different places in order to find out three different sizes of objects. In the second stage, the Pneumatic pusher will push the object. Here three pushers are used to push the objects, which will operate based on the size of the object. Whenever the object is detected then solenoid valve will be opened automatically and then the Pneumatic power will be released through valve to actuator named as pusher. Quantity of the objects in the field is sensed by the IR sensors and fed as inputs to the $Q$ series PLC which in turn are sent to system through Ethernet for necessary processing steps. A PLC program will be developed based on the signals received from the input module of PLC and the signals to the field devices from output modules of PLC, which are interfaced with the PLC output modules.
\end{abstract}

Index Terms: IR detectors, solenoid valve, SCADA, PLC and pushers.

\section{INTRODUCTION}

Pneumatic Power Based Object Sorting System is really sophisticated, This can be designed by the hardware equipment with digital control equipment i.e. PLC [8]. The methods of sequential controlling process have been changed promptly in recent years [1]. Automatic Pneumatic Power Based Object Sorting System is an important application in pharmacy, chemical, Food/ Beverage, Metal, Mining, Power, Textile, Petrochemical, Machine Manufacturing and Automobile industries. The main theme of the project follows two sequential stages.

\section{Detecting the size of the object.}

2. Pushing the object through pneumatic power.

A. Detecting the size of the object: Initially when the motor switch is turned on then conveyor belt starts running and the objects present on the belt will be detected by the proximity sensors Signals from the sensors are given to the
PLC input module terminals. Based on the customization there are three different sensors and their respective sizes of the object .According to the ladder logic program large sensor detects only large sized objects; similarly medium sized and small sensor detects small sized objects and also gives the total count of the objects. Here IR detector is used to detect the size of the bottle. A photoelectric switch, or photo eye, is a device used to detect the distance, absence, or presence of an object by using a light transmitter, often infrared, and a photoelectric receiver [7]. They are used extensively in industrial manufacturing. There are three different functional types: opposed (through beam), retro-reflective, and proximity-sensing (diffused).

B. Pushes the object through pneumatic power: Actuator is named as pusher. Whenever object is detected then PLC $\mathrm{CPU}$ sends the command signal to respective output terminal then the device is actuated which is interfaced to respected output terminal. Here the output terminal is connected to the solenoid valve [1]. Once the output terminal status is Logic 1, then the solenoid valve will be energized valve will be opened, Pneumatic force will be applied to the actuator, and then finally actuator pushes the respective object on to the outer conveyor.

The paper is ordered as follows .Segment I, Introduction to object size detection and pushing the object through pneumatic power \& techniques used to operate actuator through pneumatic power.

Segment II: Project Block diagram

Segment III: Input, output devices \& signal conditioning circuits

Segment IV: Mitsubishi PLC

Segment V: Programming language \& results

Segment VI: Conclusion and future scope

Segment VII: confession

Segment VIII: references 


\section{Block Diagram OF The Application}

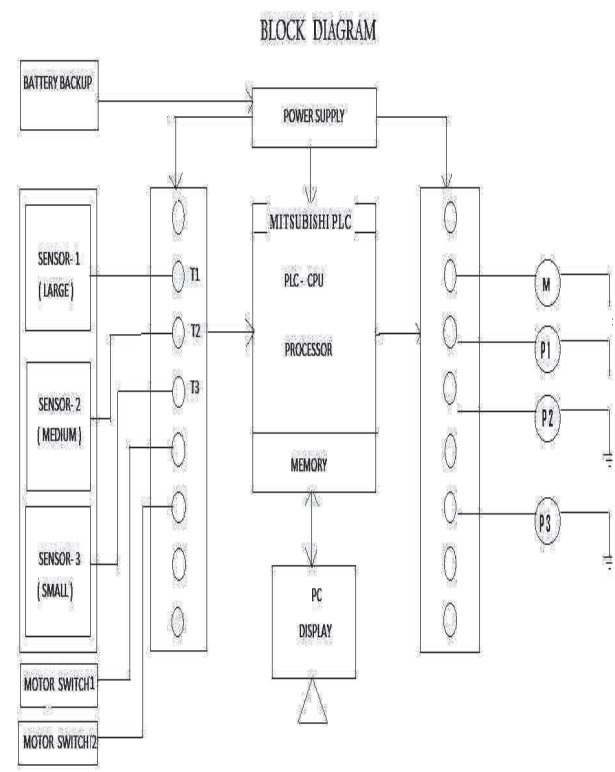

Fig 1.Block diagram

The block diagram describes the interfacing of field devices to the Programmable Logic Controllers. Here we are using Mitsubishi PLC [5]. This is a modular type of PLC. This is in the structure of slots. Each slot represents either input module or output module. In this project totally five input devices \& five output devices are used. Five input devices are master start, master stop, small object sensor, medium object sensor \& large object sensor. Output devices are indicating light, conveyor motor, Pusher1, pusher2 \& pusger3.The following table.1 represents the addressing formatting of respective terminal connections.

\begin{tabular}{|c|l|c|c|}
\hline S.No & $\begin{array}{c}\text { Name of the } \\
\text { device }\end{array}$ & $\begin{array}{c}\text { Type of the } \\
\text { device }\end{array}$ & $\begin{array}{c}\text { Addressing } \\
\text { format } \\
\text { respective } \\
\text { terminal }\end{array}$ \\
\hline 1 & master start, & INPUT & X0 \\
\hline 2 & master stop & INPUT & X1 \\
\hline 3 & $\begin{array}{l}\text { small object } \\
\text { sensor }\end{array}$ & INPUT & X2 \\
\hline 4 & $\begin{array}{l}\text { medium object } \\
\text { sensor }\end{array}$ & INPUT & X3 \\
\hline 5 & $\begin{array}{l}\text { large object } \\
\text { sensor }\end{array}$ & INPUT & X4 \\
\hline 6 & conveyor motor & OUTPUT & Y20 \\
\hline 7 & Pusher1 & OUTPUT & Y21 \\
\hline 8 & pusher2 & OUTPUT & Y22 \\
\hline 9 & pusher3 & OUTPUT & Y23 \\
\hline
\end{tabular}

Table.1 Addresses of I/O terminals

Sensor output is in the range of mvolts, and so requires signal conditioning circuits[4].PLC input modules support particular voltage \& current specifications so interfacing sensor output signal should meet the specifications of input modules.PLC out modules requires device driver circuits to drive the output devices which are connected to PLC output terminals.

Whenever master start switch is pressed then conveyor is on, next optical sensors detect the size of the bottle [1]. If the size of the bottle is medium, then the respective actuator is on. Once the object is detected, then the PLC CPU sends the control signals to the respective terminal then automatically solenoid valve will be opened and then Pneumatic signal is released through valve, which is applied to object then object is forced by Pneumatic signal. Pneumatic signal can be provided by compressor through pressure regulator [3].

\section{III- Input, Output Devices \& Signal Conditioning Circuits}

In this project five input devices $\&$ three output devices are used. Some devices requires signal conditioning circuits \& device drivers. The following devices are used to implement this project

Digital I/O: Common digital field input devices include pushbuttons, limit switches, photo sensor etc. Common digital output devices include relays, motor starters, and solenoid valves.

IR detector: This sensor is used to detect the presence of the bottle on conveyor and detect quantity of the bottle. This is shown in Fig.2. IR transmitter \& receiver circuits are used to identify the size of the object.IR transmitter always process IR signals that will fall on LDR (Light Dependent Resistor) while there is no object in-between IR transmitter and receiver [7]. Once the object will come inbetween IR source and IR destination then signal is interrupted by the object. The output of sensor is Logic ' 0 '. If the object is not in-between that, then light will fall on the LDR then, output will be logic ' 1 '

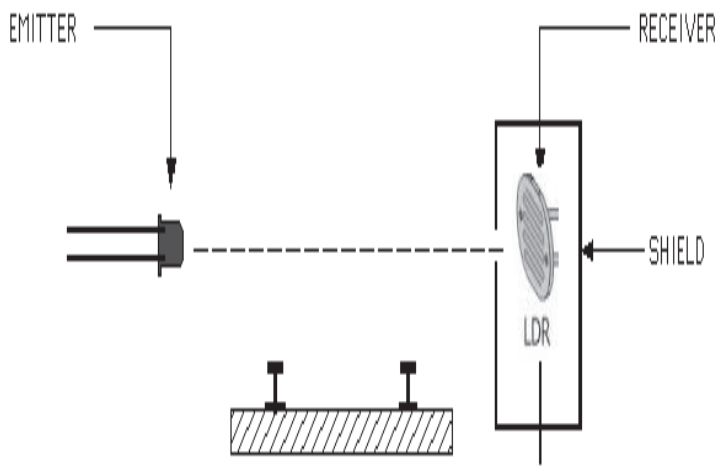

Fig.2. Proximity sensor using LDR

We fixed a light emitter LED on one point and a photo resistor on another so that, when the obstacle passes between the two points, light intensity received by the photo resistor will drop causing the resistance across it to become higher. Here output of the sensor is low voltage signal, to make the interface of this sensor to the PLC 
input modules, these signal should be amplified in order to reach the specifications of I/O modules[7] [5].

Solenoid Valve: Solenoid valve drives the Pneumatic actuator or pusher. Whenever the input of solenoid valve will be logic 1 then valve will be opened automatically then Pneumatic signal will be released to actuator then which pushes the respective object [5]. The key to the operation of a proportional valve is a balance established between the forces in action on the plunger. These balanced forces include a mechanical force provided by a spring specially developed for proportional valves and a magnetic force created by the current level passing through the coil. The spring force is proportionally opposed by the magnetic force, which is shown in Fig.3.

1. Coil

2. Spring

3. Plunger

4 .Magnetic field

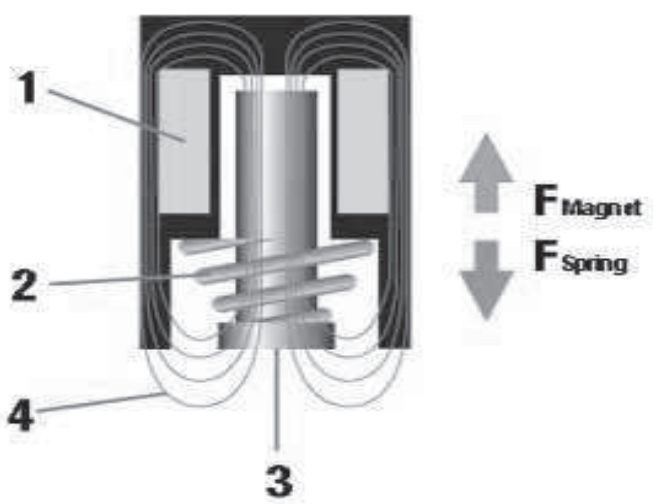

Fig.3.Soliniod valve

Solenoid valve is operated by voltage (or) Current form the PLC output modules. Solenoid valve provides the pneumatic signal to push the selected object In this project three solenoid valves are used to push three different objects from the conveyer belt pneumatic. Energy is used to push the objects here. We are providing 60psi to $80 \mathrm{psi}$ pressure to pusher. The selected objects, once the object is detected then the pusher solenoid valve will be energized, then it will be opened the pressure will be applied to object.

Signal Conditioning Circuit: output of the sensor is in the range of mvolts or volts. This voltage is not enough to interface with PLC input terminals. Which supports 14 Volts to 24 Volts range of signals but sensor produces 1.7 Volts of signal [4]. So signal conditioning circuit is required is required, here circuit is designed with operational amplifier. In view of this $\mathrm{Rf}$ can be considered as $10 \mathrm{~K}$ and Rin can be considered as $1 \mathrm{k}$.Signal conditioning circuit is shown in Fig.4.

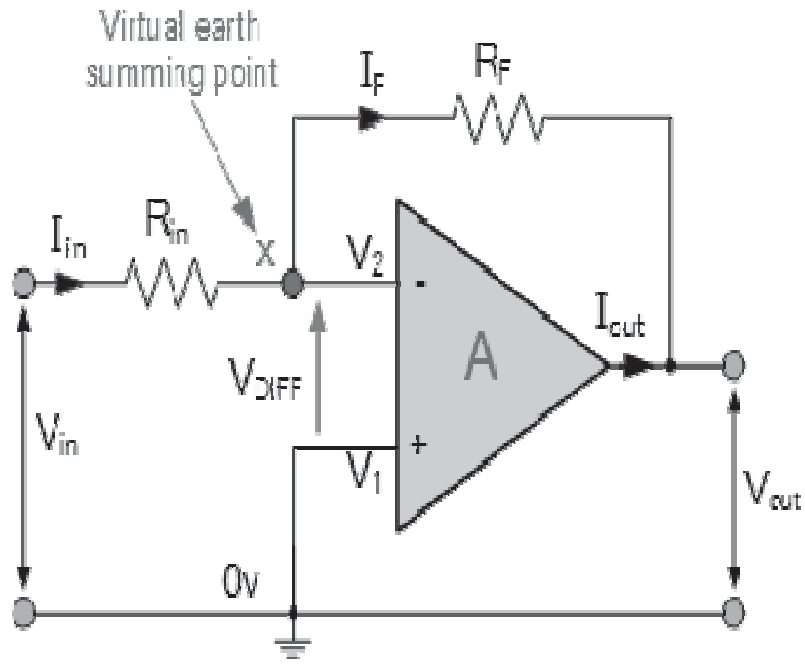

Fig.4.Signal conditioning circuit

Signal conditioning circuit is used to get desired voltage application to meets the specifications of PLC input module .In this paper we are using IR sensors to detect the objects moving on the conveyor belt. Once the object is detected by the IR senor then it produces the low voltage signal [4]. This signal to be amplified to meet the specifications of PLC input modules [4]. Which is shown in Fig.4 Input voltage $V_{i}$ is taken from the IR sensor. When the object is detected then it produces $1.2 \mathrm{~V}$ voltage, this valve should be amplified to above $10 \mathrm{~V}$. So we designed 10 gain amplifier, here we select $R_{f}=10 K \Omega$ and $R_{i=1} / K \Omega$. $R_{f}$ is the feedback resistance and $R_{i}$ is the input resistance. Finally $\mathrm{V}_{\text {out }}$ is given to input module terminal of PLC.Which meets the specifications of PLC input modules

$$
\mathrm{I}=\left(\mathrm{V}_{\text {in }}-\mathrm{V}_{\text {out }}\right) /\left(\mathrm{R}_{\text {in }}+\mathrm{R}_{\mathrm{f}}\right)
$$

Therefore, $\mathrm{i}=\left(\mathrm{V}_{\mathrm{in}}-\mathrm{V}_{2}\right) / \mathrm{R}_{\text {in }}$

$=\left(\mathrm{V}_{2}-\mathrm{V}_{\text {out }}\right) / \mathrm{R}_{\mathrm{f}}$

$\mathrm{I}=\left(\mathrm{V}_{\text {in }} / \mathrm{R}_{\text {in }}\right)-\left(\mathrm{V}_{2} / \mathrm{R}_{\text {in }}\right)$

$=\left(V_{2} / R_{f}\right)-\left(V_{\text {out }} / R_{f}\right)$,

So ,

$\mathrm{V}_{\text {in }} / \mathrm{R}_{\text {in }}=\mathrm{V}_{2}\left[1 / \mathrm{R}_{\text {in }}+1 / \mathrm{R}_{\mathrm{f}}\right]-\mathrm{V}_{\text {out }} / \mathrm{R}_{\mathrm{f}}$

And as, $\mathrm{i}=\left(\mathrm{V}_{\mathrm{in}}-0\right) / \mathrm{R}_{\mathrm{in}}$

$$
=0-\mathrm{V}_{\text {out }} / \mathrm{R}_{\mathrm{f}}
$$

The closed loop gain (Av) is given as

$$
\begin{aligned}
A_{v}= & V_{\text {out }} / V_{\text {in }} \\
& =-R_{f} / R_{\text {in }}
\end{aligned}
$$

Design of Conveyor belt: In this project conveyor belt is used for carrying of objects. This is driven by DC motor. Conveyor belt along with the sensors is shown in Fig.5. In this project conveyor belt was designed by using plastic 
material. This setup was made up of iron material the length of the conveyor belt is attached with a piece pole to arrange object detecting sensors in the proper manner. Rate of the product can be adjusted by adjusting the speed of the conveyor belt [8]. For industrial applications, different model of conveyor belts are used based on type of product [6]. Conveyor belt can be controlled perfectly by servo drivers or variable frequency drivers but in this project speed of the conveyor motor can be controlled by adjusting the voltage levels.

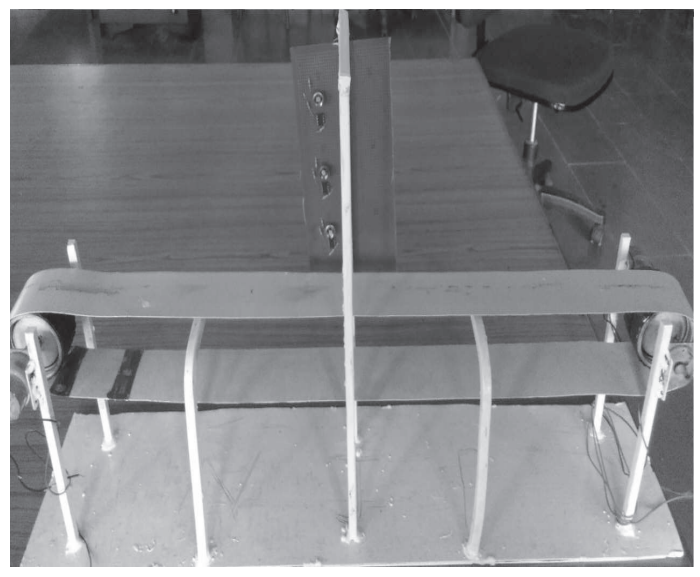

Fig.5. Conveyor belt

The belt consists of one or more layers of material are shown in Fig.5. They can be made out of plastic. Many belts in general material handling have two layers. An under layer of material to provide linear strength and shape called a carcass and an over layer called the cover. The carcass is often a woven fabric having a warp \& weft. Here we are using the material called plastic sleeve. The cover is often various rubber or plastic compounds specified by use of the belt [1]. Covers can be made from more exotic materials for unusual applications such as silicone for heat or gum rubber when traction is essential.

Here the conveyor belt will be driven by two dc motors. These two motors follow the similar direction of rotation. In the industrial applications instead of DC motors three phase induction motors are used, which are controlled by servo systems. These are $230 \mathrm{~V}$ or $440 \mathrm{~V}$ ranges of electrical motors are used. Industries follows different structure of conveyor belts depends of the application of the process. In this project we designed conveyor using plastic material

\section{Mitsubishi PLC}

In this project automation is done by aided of hardware with the PLC (Programmable Logic controller).Here Mitsubishi Q series modular PLC is used, which programming is easy to write $\&$ edit. This consumes less power.

In this project we are using PLC ladder programming supporting software is MELSOFT GX Works-2.In this paper we are using basic PLC instructions, counter and timer functions. This PLC basically has input and an output module to make the proper connection of field devices [3].This application emulates the industrial applications. In this project we are using three object sensors, four conveyor motors and master start and stop buttons. All these field devices interfaced in proper way.

Now a day's all the industries are getting automation through PLC and SCADA systems [5]. Different manufacturers are available from the world. Whatever manufacturer's .we should follow their specifications and carefully give the connections by considering sinking and sourcing in PLC [3][5]. Otherwise it causes to flow of wrong direction of current then it PLC will get damage.

Different types of PLC are available in Mitsubishi PLC. Here we are using Modular PLC. This is shown in Fig.6.

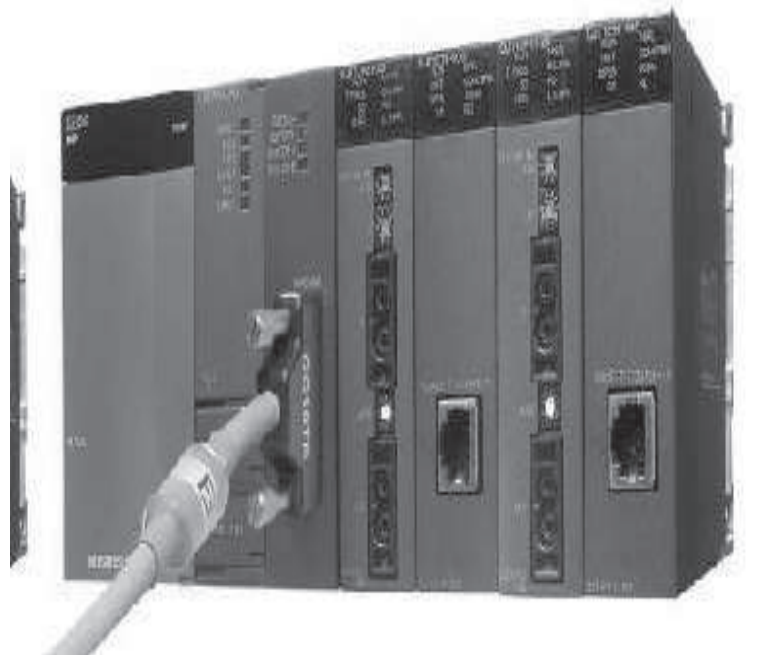

Fig.6.modular Mitsubishi PLC

MELSEC-Q Series: MELSEC-Q series: It is a modular type of PLC, in this PLC number of the modules can be extended by our requirement. It has a facility to make the communication with CC-Link and also have remote I/O modules [5] .It has high speed of the operation and different series and model of Q PLC are available with innovative specifications. Seven types of power supply units are available according to different input/output voltages, and output current values.

Different power supply units of PLC are shown in table.2.In this project we are using Q61P power supply unit.

TABLE. 2 .

DifFERENT POWER SUPPLy MOdULES OF PLC.

Digital I/O Module: These are used to interface the input and output field devices. In order to make interfacing of field devices, we need to meet the voltage \& current specifications of PLC I/O modules [3]. These are discussed over here.

Digital input module: The following table. 3 gives you an overview of different types on digital input modules 
with specifications. These specifications are given in the form of module name, points, input voltage, and sink/source type and response time of the specific module. In this project we are using QX81 input module [5].

Digital input module: QX

- Electrically isolated with opto couplers

- Removable terminal blocks for 16 connections addresses

- D-sub or 40-pin plug connector for 32 or 64 addresses

TABLE3.

DIFFERENT INPUT MODULES \& THEIR POWER RATINGS

\begin{tabular}{|c|c|c|c|l|}
\hline Module & Points & Input Voltage & Sink/Source & Response Time \\
\hline QX10 & 16 & $100-120 \mathrm{VAC}$ & - & $20 \mathrm{~ms}$ \\
\hline QX28 & 8 & $240 \mathrm{VAC}$ & & $20 \mathrm{~ms}$ \\
\hline QX40 & 16 & $24 \mathrm{VDC}$ & Sink & $1 / 5 / 10 / 20 / 70 \mathrm{~ms}$ \\
\hline QX40-S1 & 16 & $24 \mathrm{VDC}$ & Sink & $0.1 / 2 / .4 / .6 / 1 \mathrm{~ms}$ \\
\hline QX41 & 32 & $24 \mathrm{VDC}$ & Sink & $1 / 5 / 10 / 20 / 70 \mathrm{~ms}$ \\
\hline QX41-S1 & 32 & $24 \mathrm{VDC}$ & Sink & $0.1 / 2 / .4 / .6 / 1 \mathrm{~ms}$ \\
\hline QX42 & 64 & $24 \mathrm{VDC}$ & Sink & $1 / 5 / 10 / 20 / 70 \mathrm{~ms}$ \\
\hline QX70 & 16 & $5 / 12 \mathrm{VDC}$ & Sink/Source & $1 / 5 / 10 / 20 / 70 \mathrm{~ms}$ \\
\hline QX71 & 32 & $5 / 12 \mathrm{VDC}$ & Sink/Source & $1 / 5 / 10 / 20 / 70 \mathrm{~ms}$ \\
\hline QX72 & 64 & $5 / 12 \mathrm{VDC}$ & Sink/Source & $1 / 5 / 10 / 20 / 70 \mathrm{~ms}$ \\
\hline QX80 & 16 & $24 \mathrm{VDC}$ & Source & $1 / 5 / 10 / 20 / 70 \mathrm{~ms}$ \\
\hline QX81 & 32 & $24 \mathrm{VDC}$ & Source & $1 / 5 / 10 / 20 / 70 \mathrm{~ms}$ \\
\hline
\end{tabular}

Digital output module: This digital output module table .4 given below gives different module names and their specifications in the form of module name, points, output voltage, device which can be driven, current, points/com etc [5]. In this project we are using QY41P output module. Digital output module: QY

- Module with relay, transistor or triac output technology

- Electrical isolations of process /control and also channel in some Cases

- Choice of interface modules.

\section{TABLE.4.}

DifFerent OUtPut Modules \& THEIR POWER RATINGS

\begin{tabular}{|c|c|c|c|c|c|}
\hline Module & Points & Op Voltage & Device & curentopint & Points/Com \\
\hline QY10 & 16 & ZLWVACLLVDC & Reay & 24 & 16 \\
\hline QY18A & 8 & ZLOVACLLVOC & Reay & 24 & 1 \\
\hline QY4Q01* & 16 & $12 / 24 \sqrt{0 C}$ & Trens stor & $0.2 A$ & 16 \\
\hline QYAIP & 32 & $12 / 24 V 06$ & Irens stor & 0.14 & 32 \\
\hline QY42P & 64 & $12 / 24 \sqrt{ } 06$ & Irens stor & $0.12 A$ & 32 \\
\hline QYsu & 16 & $12 / 24 \sqrt{06}$ & Ireris stor & 0.54 & 16 \\
\hline ar2 & 16 & 240VAC & Iriac & $0.6 A$ & 16 \\
\hline QYro & 16 & $5 / 12 V D C$ & Inensistor & $16 m A$ & 16 \\
\hline QY71 & 32 & $5 / 12 v 0 \mathrm{C}$ & Innsistor & $0.026 \mathrm{~A}$ & 32 \\
\hline 4480 & 16 & $12 / 2480 \mathrm{C}$ & Irens stor & $0.5 A$ & 16 \\
\hline Qrsip & 32 & $12 / 24 v 00$ & Trensistor & $0.1 . A$ & 32 \\
\hline QY688 & 8 & $y-24 v 0 C$ & Irasistor & 24 & 1 \\
\hline
\end{tabular}

\section{Programming Language \& Results}

Programming of PLC using GXWorks2: GX Works consists of various different components that help to simplify project creation and maintenance tasks [5]. A system design console that enables projects to be created at the system overview stage has been added. Additionally, the main programming languages are supported and their labels (variables) are shared, further simplifying programming. Various debug and maintenance features are also included.

\section{Addressing In Q-PLC:}

- Addressing is in Hexadecimal

- $\quad$ Each module is of fixed no. of I/O points i.e. 16, 32,64 etc.

- $\quad$ Each module takes Input points and output points from the image memory.

- Usage of allotted I/O points depends on the type of module.

- Example: QX81 is 32 point module, uses only input points.

- Flexible: Any card can be installed in any slots.

No Jumpers setting/DIP setting on modules.

I/O Addressing: The following Fig.7 represents the addressing formats of the I/O modules. PLCs may follow hexa, octal or binary addressing formats and is prescribed by the manufacturer [5][3]. Most of them use hexadecimal format of addressing and so as we use that here. The following are the tables which represent how the addressing is made to the particular slot of the memory. 


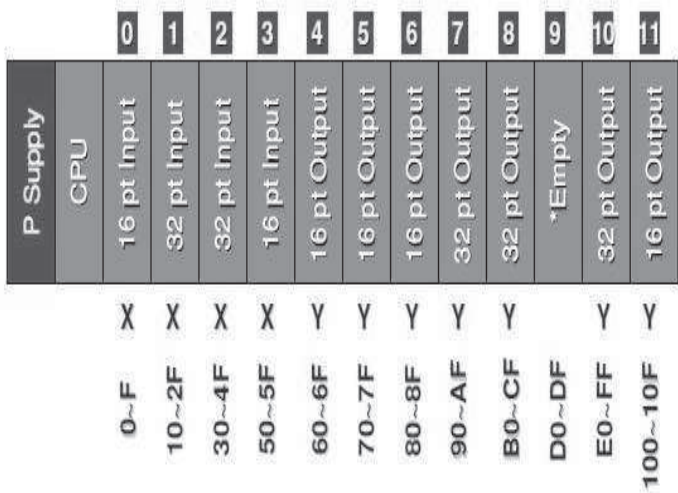

Fig .7.Addressing Formatting Of I/O Modules

The following Instructions are required to implement PLC programming through $\boldsymbol{G} \boldsymbol{X}$ Works 2

Input $(\mathbf{X})$ : Inputs are designed to give command and data from external devices, e.g. pushbuttons, select switch, etc., to the PLC. Inputs are available as "refer Inputs" and "direct access inputs" [5].

Output (Y): Outputs are used to provide the control results of a program from the PLC external devices, e.g. solenoid, signal lamps, etc. Outputs are available as "refresh outputs" and "direct access output".

Internal relays $(\mathbf{M})$ :Internal relay are auxiliary relays used in a CPU module and not latched.

Latch relays $(\mathbf{L})$ : Latch-relays are auxiliary relays used in a CPU module and latched (backed up at power failure).

Timer (T): A timer is a device that starts counting when its coil turns $\mathrm{ON}$, and timer-out and turns $\mathrm{ON}$ its contact when the current value reaches or exceeds the set value. The timer is of an up-counting type. The current matches the set value when a "time-out" occurs [5].

Counter (C): A counter is a device which counts the number pf inputs condition edges in sequence programs. When the counter value matches the value, its contact turns ON. The counter is of an up-counting.

Ladder Programming: This is the source code of our project. This is the ladder programming which was simulated and tested many times in GXWorks2 software which is compatible with Mitsubishi Q-PLC. In this project if the objecte is detected, then the respective pusher will be on after some time delay [3]. Here time delay can be maintained by PLC timer function where base time of the timer to be set. Setting the Base time of the timer is shown in Fig.9.therefore when the below program Fig.8 is written in GXWorks2 software and simulated using the Mitsubishi Q-PLC [5] by connecting the PC and PLC with an Ethernet port, the PLC actuates the inputs and outputs connected to it according to the program written is shown in Fig.9. In this way, we were able to visualize our output which was according to what we have planned.

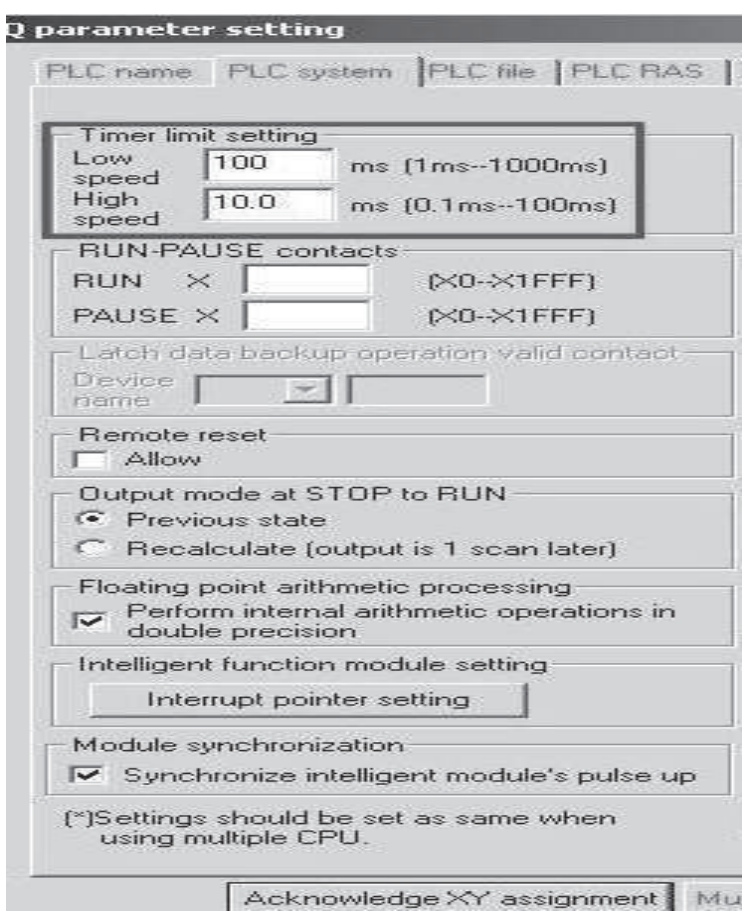

Fig.8.Timer limit setting
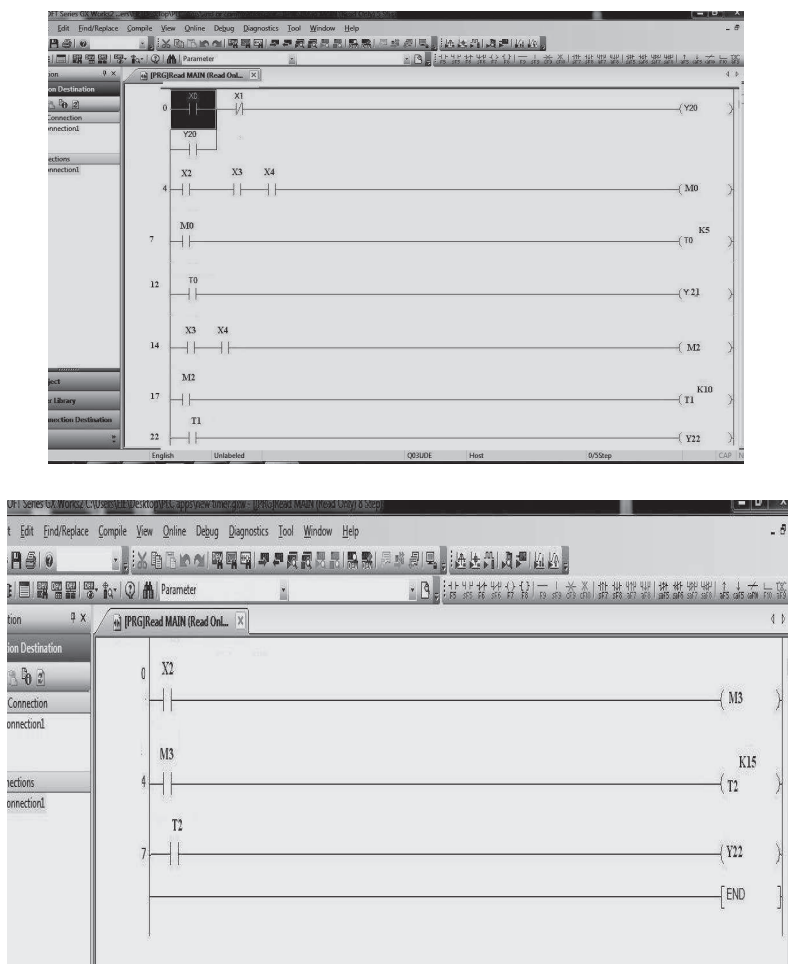

VI.CONCLUSION

The automation technique proposed in this paper is very useful in industries where there are many parameters apart from those we considered in this experiment, which can be automatically controlled by Q-PLCs. This lessens the human efforts and errors caused by human beings when conducting this process manually. 


\section{REFERENCES}

[1]. Bateson, N.R. "Introduction to Control System Technology", Sixth Edition, United State of America: Prentice Hall.1999

[2]. Vitthal G. Chikte 1, Priyanka D. Ughade 2 "Industrial Automation Of Object Sorting System Using Programmable Logic Controller (PLC)" International Journal For Engineering Applications And Technology. ISSN: 2321-8134. Volume 2 No 9 September 2012.

[3]. John. W .Webb Ronald A Reis, Programmable Logic Controllers - Principles and Applications, Fourth edition, Prentice Hall Inc., New Jersey, 1998.

[4]. D. Roy choudary,Jain, Shail B "Linear Integrated Circuits", Fourth Edition, New age International Publishers.2006.

[5]. http://www.mitsubishielectric.com/fa/assist/elearning/\#page01.

[6]. M.Z.A Rashid1, H.N.M Shah1, H.I Jaafar1, M.S.M Aras1 and S.K.S Nordin2 "Sorting and Retrieval Robotic System Controlled via Programmable Logic Controller for Library Usage". International Journal of u-and eService, Science and Technology Vol.7, No.4 (2014), pp.19-30

[7]. Lasers and Optical Engineering - by Das P., Springer's International Students Edition, 1991.

[8]. G. Sujatha1 and V.Perasiriyan "Conveyor Control and Sorting Module Controlled by Programmable Logic Controller" International Journal of Applied Engineering and Technology ISSN: 2277-212X (Online) An Open Access 2014 Vol. 4 (2) April-June, pp.12-18. 